\title{
Redispersible spray-dried lipid-core nanocapsules intended for oral delivery: the influence of the particle number on redispersibility
}

Diego Fontana de Andrade, Branko Vukosavljevic, ${ }^{\mathrm{c}, \mathrm{d}}$, Edilson Valmir Benvenutti ${ }^{\mathrm{b}}$, Adriana Raffin Pohlmann ${ }^{\mathrm{a}}$, Sílvia Stanisçuaski Guterres ${ }^{\mathrm{a}}$, Maike-Windbergs ${ }^{\mathrm{c}, \mathrm{d}}$, Ruy Carlos Ruver Beck ${ }^{\mathrm{a}^{*}}$

aPrograma de Pós-Graduação em Ciências Farmacêuticas, Universidade Federal do Rio Grande do Sul, Porto Alegre, RS, Brazil. ${ }^{b}$ Departamento de Química Orgânica, Instituto de Quimica, Universidade Federal do Rio Grande do Sul, Porto Alegre, RS, Brazil. 'Saarland University, Department of Biopharmaceutics and Pharmaceutical Technology, Saarbruecken, Germany. ${ }^{d}$ Helmholtz Centre for Infection Research (HZI) and Helmholtz Institute for Pharmaceutical Research Saarland (HIPS), Department of Drug Delivery, Saarbruecken, Germany.

* Author to whom correspondence should be addressed: Prof PhD R.C.R. Beck; Departamento de Produção e Controle de Medicamentos, Universidade Federal do Rio Grande do Sul, Avenida Ipiranga, 2952, 90610-000, Porto Alegre, RS, Brazil; Phone +55 51 33085215; E-mail: ruy.beck@ufrgs.br 


\section{Redispersible spray-dried lipid-core nanocapsules intended for oral delivery: the influence of the particle number on redispersibility}

This study proposes a new approach to produce easily redispersible spray-dried lipid-core nanocapsules (LNC) intended for oral administration, evaluating the influence of the particle number density of the fed sample. The proposed approach to develop redispersible spray-dried LNC formulations intended for oral route is innovative, evidencing the needing of an optimization of the initial particle number density in the liquid suspension of nanocapsules. A mixture of maltodextrin and L-leucine $(90: 10 \mathrm{w} / \mathrm{w})$ was used as drying adjuvant. Dynamic light scattering, turbidimetry, determination of surface area and pore size distribution, electron microscopy and confocal raman microscopy (CRM) were used to characterize the proposed system and to better understand the differences in the redispersion behavior. An easily aqueous redispersion of the spray-dried powder composed of maltodextrin and L-leucine $(90: 10 \mathrm{w} / \mathrm{w})$ was obtained, depending on the particle number density. Their surface area decreased in the presence of LNC. CRM enabled the visualization of the spatial distribution of the different compounds in the powders affording to better understand the influence of the particle number density of the fed sample on their redispersion behavior. This study shows the need for optimizing initial particle number density in the liquid formulation to develop redispersible spray-dried LNC powders.

Keywords: drug delivery, L-leucine; maltodextrin; lipid-core nanocapsules; spray-drying. 


\section{Introduction}

In the course of finding novel effective therapeutics, numerous new nanosized carrier systems for drug delivery have been developed, among which sophisticated systems such as polymeric nanocapsules. These nanocapsules form vesicular structures containing an oily core surrounded by a polymeric shell. Furthermore, recently lipidcore nanocapsules (LNC) were reported, which are single core structures prepared by interfacial deposition of preformed polymer. Their core is composed of an organogel formed by a combination of liquid and solid lipids enveloped by a polymer. Their composition give them distinctive mechanical properties, when compared to polymeric nanocapsules containing only a liquid lipid core. (Fiel et al. 2011, Venturini et al. 2011, Rigo et al. 2014).

The production, characterization, and biological properties of LNC have been the object of study by our research group. Some of the main advantages of drug-loaded LNC certainly point to their potential to increase drug bioavailability (Dimer et al. 2014, Friedrich et al. 2014, Frozza et al. 2013) and to improve antitumor activity (Figueiro et al. 2013). In addition, LNC have gastroprotective effect (Bueno et al. 2013) and the ability to modulate drug release through different skin layers (Fontana et al. 2011).

In order to preserve and maintain the physicochemical and microbiological stability of these nanocapsules, the usual approach comprises the conversion of the aqueous dispersions into solid systems (Okuyama et al. 2006, Schaffazick et al. 2003). Dry powders prepared from nanocarriers represent an important breakthrough in the development of different solid dosage forms designed for different administration routes such as the oral (Morgen et al. 2012, Ozeki et al. 2006, Pamujula et al. 2004), 
intramuscular (Muttil et al. 2010), and pulmonary routes ADDIN EN.CITE.DATA(Ely et al. 2007, Mohajel et al. 2012, Ungaro et al. 2012).

Freeze-drying (Abdewahed et al. 2006) and spray-drying (Hoffmeister et al. 2012, Muller et al. 2000, Schaffazick et al. 2006, Guterres et al. 2006, Tewa-Tagne et al. 2007) have been reported as suitable techniques to convert nanosuspensions, as polymeric nanocapsules, into solid systems, along with the wet granulation (Friedrich et al., 2010) and 3D printing techniques (Beck et al., 2017). Due to the complex vesicular character and the oily core of the nanocapsules, freeze-drying is not the easiest process for this purpose (Chasteigner et al. 1996, Pohlmann et al. 2002). In this scenario, spraydrying and nano spray-drying have been widely studied for this conversion (Guterres et al., 2009; Arpagaus et al. 2017). Moreover, in order to preserve these systems during the spray-drying procedure, different drying adjuvants have been utilized as structural support (Beck et al. 2007, Hoffmeister et al. 2012, Schaffazick et al. 2006, Tewa-Tagne et al. 2007). This technique has been also employed in the production of microparticulate systems containing nanoparticles (El-Sherbiny and Smyth 2010, Lebhardt et al. 2011, Li et al. 2013), as it allows rapid and continuous processing, providing high batch-to-batch reproducibility.

However, many biological advantages of LNC are attributed to their physical and surface characteristics (particularly particle size and surface area), so rapid and easy aqueous redispersion of nanoparticles and the subsequent protection of their integrity are of great importance. Even though individual approaches like the use of hydrophilic adjuvants, such as lactose, mannitol, and dextrin (Hoffmeister et al. 2012, Sham et al. 2004, Tewa-Tagne et al. 2007), highly diluted nanostructured systems (Tewa-Tagne et al. 2007), as well as effervescent systems (Ely et al. 2007) are as strategies to improve the process of redispersion of nanocarriers from their dry powders, there is a lack of 
information about the definition and standardization of analytical methods that if combined are able to provide substantial information about the redispersion efficiency.

When the oral route is the administration pathway, nanocapsules have to be transformed or embedded into an appropriate dosage form for swallowing. Further, upon arrival in the gastrointestinal tract, this dosage form should redisperse into the initial nanocarriers in order to provide the desired advantages afforded by their nanosize. Taking these facts into account, this study proposes a strategy to produce easily redispersible dry powders containing LNC as a promising pharmaceutical dosage form. The main focus of the experimental design was based on investigating and evaluating the influence of nanocapsule concentration on the efficiency of particle redispersion. Furthermore, the physicochemical properties of redispersed spray-dried LNC were analyzed in detail, using a combination of techniques such as laser diffraction, scanning electron microscopy, and confocal Raman microscopy.

\section{Materials and methods}

\section{Materials}

Poly( $\varepsilon$-caprolactone) $(\mathrm{PCL})(\mathrm{Mw}=80,000)$ and sorbitan monostearate were purchased from Sigma-Aldrich (São Paulo, Brazil). Grape seed oil was supplied by Delaware (Porto Alegre, Brazil). Polysorbate 80 was acquired from Henrifarma (São Paulo, Brazil). Acetone was obtained from Nuclear (São Paulo, Brazil). Maltodextrin (dextrose equivalent $(\mathrm{DE})=19)$ and L-leucine were purchased from Roquette (Lestrem, France) and Fagron (São Paulo, Brazil), respectively. 


\section{Preparation and characterization of the lipid-core nanocapsule suspension}

The lipid-core nanocapsules (LNC) were prepared based on the interfacial deposition of a preformed polymer (Jaques et al. 2013, Venturini et al. 2011, ZanottoFilho et al. 2013) as liquid intermediate products. Firstly, an organic phase was prepared dissolving Poly( $\varepsilon$-caprolactone) (0.5 g), sorbitan monoestearate (0.19 g) and grape seed oil $(825 \mu \mathrm{L})$ in acetone $(135 \mathrm{~mL})$ at $40{ }^{\circ} \mathrm{C}$. After the complete solubilization of the components, the organic solution was injected into an aqueous solution $(270 \mathrm{~mL})$ containing polysorbate $80(0.14 \%)$ under magnetic stirring. Lastly, acetone and water were evaporated under reduced pressure to a final volume of $50 \mathrm{~mL}$. Triplicate of batches were prepared and stored at room temperature, protected from light, upon analysis.

In order to ensure the particle size distribution of the LNC only at the nanoscale, these formulations were evaluated by laser diffraction (Mastersizer $^{\circledR}$ 2000, Malvern Instruments, Malvern, UK). In addition, their mean size and polydispersity index (PDI) were determined by dynamic light scattering (Zetasizer Nano $\mathrm{ZS}^{\circledR}$, Malvern Instruments, Malvern, UK) at $25{ }^{\circ} \mathrm{C}$ after previous dilution of LNC suspension in ultrapure water $(500 \mathrm{x})$. Zeta potential was measured by electrophoretic mobility (Zetasizer Nano $\mathrm{ZS}^{\circledR}$, Malvern Instruments, UK) after previous dilution of LNC suspension in $10 \mathrm{mmol} \mathrm{L}^{-1} \mathrm{NaCl}$ aqueous solution $(500 \mathrm{x})$. 


\section{Analysis of particle number density}

To estimate the particle number density of the LNC suspension, a turbidimetric method was used (Zattoni et al. 2003). Turbidity affords to estimate the number of particles per $\mathrm{mL}$ in LNC suspensions (Poletto et al. 2008). Initially, the UV-visible wavelength where absorption is minimum was determined, in order to avoid any photon absorption by the material and to guarantee that the scattering is the only phenomenon of light and matter interaction due to the presence of colloids in solution. In this study, the wavelength of $397 \mathrm{~nm}$ was chosen after an experimental optimization (data not shown). After this step, the LNC suspension was diluted in ultrapure water in a range designed so as to obey the Lamber-Beer law $(2,3,4,5$ and $6 \mu \mathrm{L}$ of formulation per $\mathrm{mL}$ of ultrapure water). Samples $(n=3)$ were analyzed using a spectrophotometer (Shimadzu® UV-1800PC, Shimadzu, Japan).

The turbidity $(\tau)$ was calculated as a function of the absorbance A, according to Eq. (1):

$\tau=\frac{1}{b} \ln (10) . A$

where $\mathrm{b}(\mathrm{cm})$ represents the optical cell path length and $\mathrm{A}$ is the instrumental absorbance signal.

Turbidity was calculated for each concentration of samples.

Furthermore, the particle number density of LNC suspension (particles $\mathrm{cm}^{-3}$ ) was obtained as a function of $\tau$ according Eq. (2): 
$\tau=\frac{\pi}{4} \cdot d^{2} \cdot N \cdot Q$

Moreover, Q can be obtained as the following equation (Eq. 3), at a fixed relative refractive index.

$$
Q=K .2 \ln (10) \cdot \alpha \cdot d / 3
$$

$\mathrm{d}(\mathrm{cm})$ is the particle diameter, $\alpha\left(\mathrm{g} \mathrm{cm}^{-3}\right)$ is the particle density, obtained as a ratio between the sum of the volume fractions of the components of the nanocapsules considering the individual density of each material and a total mass of solids that this volume represents. $\mathrm{K}\left(\mathrm{cm}^{2} \mathrm{~g}^{-1}\right)$ is the sample extinction coefficient, which can be calculated using the equation below (Eq. 4).

$\tau=\ln (10) . K . C$

Where $\mathrm{c}\left(\mathrm{g} \mathrm{cm}^{-3}\right)$ is the sample concentration of solids in dispersion. The sample concentration was obtained for each analyzed concentration $(2,3,4,5$ and $6 \mu \mathrm{L}$ of formulation per $\mathrm{mL}$ of ultrapure water). The diameters $\mathrm{d}$ (Eq. 3) were previously determined (section "Preparation and characterization of the lipid-core nanocapsule suspension").

\section{Preparation of spray-dried lipid-core nanocapsule powder}

Spray-dried LNC were prepared using a Buchi Mini Spray Dryer B-290 (Buchi, Switzerland) under the following conditions: nozzle with a cap orifice diameter of 0.7 
$\mathrm{mm}$, nozzle at a feed rate of $4.5 \mathrm{~mL} \mathrm{~min}^{-1}$, inlet temperature maintained at $140{ }^{\circ} \mathrm{C}$ resulting in an outlet temperature of $79{ }^{\circ} \mathrm{C}$. The drying airflow rate was $35 \mathrm{~m}^{3} \mathrm{~h}^{-1}$ (Hoffmeister et al. 2012). Maltodextrin and L-leucine were used as drying adjuvants $(90: 10 \mathrm{w} / \mathrm{w})$ at a concentration of $2.25 \%(\mathrm{w} / \mathrm{v})$ and $0.25 \%(\mathrm{w} / \mathrm{v})$ in the NC suspension, respectively. They were added under magnetic stirring at least 20 min before the drying process. Three different powders were produced: the first one containing undiluted LNC (F100), the second containing LNC diluted in water at a ratio of 3:1 (v/v) (F75:25), and the last one containing LNC diluted in water at a ratio of $1: 1(\mathrm{v} / \mathrm{v})(\mathrm{F} 50: 50)$. To produce the powders from diluted LNC (F75:25, F50:50), the mixture of adjuvants was dissolved before the spray-drying process in the specified volume of water used to dilute the LNC. After preparation, powders were stored at room temperature (20 - 25 ${ }^{\circ} \mathrm{C}$ ), in hermetically sealed amber flasks, protected from light and moisture.

The composition of these samples fed to the spray-drying process are detailed in Table 1.

INSERT TABLE 1 ABOUT HERE

Furthermore, two additional powders were produced. The first one (called as F50:50M) was produced according to the composition of the formulation F50:50, but using only maltodextrin as the drying adjuvant (omitting L-leucine). The second one, F50:50B, was produced by replacing the nanocapsules suspension by ultrapure water.

\section{Physicochemical characterization of spray-dried lipid-core nanocapsules powders}

Process yield 
Process yield was calculated as the ratio between the weight of powder recovered into the collector compartment of the spray-dryer and the sum of the weights of all components of the formulation, disregarding the water content.

Performance of the aqueous powder redispersion

Particle size distribution profiles of the redispersed powders in water were assessed by laser diffraction (Mastersizer ${ }^{\circledR}$ 2000, Malvern Instruments, UK). A small amount of each sample was added into the wet dispersion unit of the equipment containing ultrapure water, at a laser obscuration of at least $2 \%$. Measurements were carried out right after the addition of the powder into this unit.

Determination of surface area and pore size distribution

The nitrogen adsorption-desorption isotherms of the spray-dried LNC formulations (F100, F75:25, and F50:50) and F50:50B (previous degassed under vacuum at $45^{\circ} \mathrm{C}$ for $12 \mathrm{~h}$ ) were determined at liquid nitrogen boiling point using a Tristar Krypon 3020 Micromeritics surface area analyzer. The specific surface areas of powders were determined by the BET (Brunauer, Emmett and Teller) multipoint technique, and the pore size distribution was obtained using the BJH (Barret, Joyner and Halenda) method. The comparisons of the data were performed by one-way analysis of variance (ANOVA) followed by Tukey post hoc test using GraphPad Prism software version 5.01 (GraphPad Software Inc. La Jolla, CA, USA). Statistical significance was considered when $p \leq 0.05$. 


\section{Morphological characterization of the spray-dried lipid-core nanocapsules}

Scanning electron microscopy

Morphology of the spray-dried powders (F100, F75:25, and F50:50 and F50:50B) was analyzed by scanning electron microscopy (SEM) (Jeol Scanning Microscope, JSM-6060, Tokyo, Japan) at $10 \mathrm{kV}$, using different magnifications (between 1,000x and 20,000x). Samples were analyzed after gold sputtering (Jeol Jee 4B SVG-IN, Tokyo, Japan). These analyses were carried out at the Centro de Microscopia e Microanalises at the Federal University of Rio Grande do Sul (CMMUFRGS, Brazil).

Confocal Raman microscopy (CRM)

Confocal Raman microscopy (CRM) measurements were performed with a WITec alpha 300R+ (WITec GmbH, Ulm, Germany). The excitation source was a monochromatic diode laser with an excitation wavelength of $532 \mathrm{~nm}$ adjusted to a power of $20 \mathrm{~mW}$ ahead of the $50 \mathrm{x}$ magnification (N.A. 0.8) objective (Epiplan Neofluar, Zeiss, Germany). A confocal $50-\mu \mathrm{m}$ pinhole rejected signals from out-offocus regions. Raman spectra of the pure compounds were acquired with an integration time of $0.5 \mathrm{~s}$ and 10 accumulations. Image scans of the samples were recorded with an integration time of $0.5 \mathrm{~s}$ and a step size was $0.5 \mu \mathrm{m}$ along the $\mathrm{x}$ - and $\mathrm{y}$-axis. After background subtraction and normalization, spectra were converted into false color images using the WITec Project Plus software (WITec GmbH, Ulm, Germany). Image 
pixels assigned to maltodextrin and L-leucine spectra are depicted in blue, while lipidcore nanocapsules appear in yellow.

\section{Determination of redispersion efficiency}

Performance of the powder redispersion in water

Powder samples $(0.695 \mathrm{~g})$ were diluted with ultrapure water in a volumetric flask of $5 \mathrm{~mL}$ and vortexed for $2 \mathrm{~min}$ at room temperature. After, the particle size distribution profile of each dispersed medium was analyzed by laser diffraction (Mastersizer $^{\circledR}$ 2000, Malvern Instruments, Malvern, UK) according to the procedure previously described in section "Performance of the aqueous powder redispersion".

\section{Transmission electron microscopy}

The integrity of nanostructures after the spray-dried powder redispersion was observed by transmission electron microscopy (TEM) (JEM 1200 Exll, Jeol, Tokyo, Japan) operating at $80 \mathrm{kV} .0 .1 \mathrm{~mL}$ of the redispersed powder in water (according to the description in item 2.7.1) or $0.1 \mathrm{~mL}$ of the original LNC suspension was diluted in 0.9 $\mathrm{mL}$ of ultrapure water. Next, an aliquot of each sample was deposited on a specimen grid (Formvar-Carbon support films, Electron Microscopy Sciences, Hatfield, USA), negatively stained with uranyl acetate solution $(2 \%, w / v)$, and analyzed under different magnifications (between 100,000 $\times$ and 250,000 $\times$ ). These analyses were carried out at the Centro de Microscopia e Microanalises at the Federal University of Rio Grande do Sul (CMM-UFRGS, Brazil). 
Analysis of particle number density after powder redispersion

The redispersion efficiency of the spray-dried powders was evaluated comparing the particle number density of the original LNC suspension and the particle number density of each redispersed spray-dried powder. The protocol previously described in section "Analysis of particle number density" was used. For the spray-dried powder samples, the supernatant of each redispersed powder was analyzed after centrifugation at 4,500 $\mathrm{g}$ for $10 \mathrm{~min}$. The centrifugation step was performed in order to eliminate possible interferences in the analysis of particle number density after powder redispersion due to the presence of micrometric particles in suspension. The parameters were adapted from the methodology described by Zanotto and co-workers (2013) on the extraction step for determination of drug content in lipid-core nanocapsules formulation.

\section{Results}

\section{Physicochemical properties and particle density number of lipid-core nanocapsules}

LNC suspensions had monomodal particle size distribution, with mean particle size $\left(\mathrm{D}_{4.3}\right)$ of $176 \pm 1 \mathrm{~nm}$ and Span value of $1.64 \pm 0.05$, according to the laser diffraction analysis. Moreover, according to the dynamic light scattering analyses, they had a Z-average diameter of $181 \pm 1 \mathrm{~nm}$ and a polydispersity index below 0.1 . The zeta potential was negative for all batches $(-6 \pm 1 \mathrm{mV})$.

The particle number density calculated by turbidimetry was $0.775 \times 10^{13} \mathrm{LNC}$ per milliliter of formulation. In addition, their dilutions $(3: 1$ and $1: 1)$ used to fed the 
spray-drying process had a particle number of $0.581 \times 10^{13}$ and $0.387 \times 10^{13}$, for these two different dilutions, respectively.

\section{Characterization of redispersible spray-dried lipid-core nanocapsules}

Process yield and particle size distribution after water redispersion

The process yields were $64 \%$ for F50:50B, $66 \%$ for $\mathrm{F}: 100$, $68 \%$ for $\mathrm{F} 75: 25$, and $67 \%$ for F50:50 spray-dried powders. Fig. 1 presents the particle size distribution profiles of candidates for redispersible spray-dried LNC formulations and original LNC-B suspension. All spray-dried formulations presented particle size population in the micrometric range. The distribution profile of particles of formulations F100 and F75:25 differed from that of the original LNC-B suspension. On the other hand, particle size populations in the nanometric range were observed after aqueous redispersion for formulation F50:50 in water.

\section{INSERT FIGURE 1 ABOUT HERE}

The distribution profiles of particles of the spray-dried powder prepared using only maltodextrin as the drying adjuvant (Formulation F50:50M) differed from the distribution profile of the original LNC-B suspension. All particles were in the micrometric scale (Fig. 2), even after $5 \mathrm{~min}$ of stirring. Unfortunately, it was not possible to determine the particle size distribution profile by wet dispersion for the formulation F50:50B (powders prepared without nanocapsules), because the minimum optical obscuration to perform the analysis could not be reached. 
INSERT FIGURE 2 ABOUT HERE

Determination of surface area and pore size distribution

Table 2 shows the surface areas and the pore volumes of F100, F75:25, F50:50 and F50:50B spray dried powder particles according to the $\mathrm{N}_{2}$ adsorption-desorption isotherms and calculated using the BET and the BJH methods, respectively. The values of standard deviation of the analysis were below the measurement uncertainty of the method (indicated in parentheses next to each parameter name in the table). For this reason, we consider the standard deviation of the measurement uncertainty of the method as the standard deviation of each sample analyzed $\left( \pm 0.05 \mathrm{~m}^{2} \mathrm{~g}^{-1}\right.$ for BET surface area and $\pm 0.002 \mathrm{~cm}^{2} \mathrm{~g}^{-1}$ to BJH Pore volume analysis).

INSERT TABLE 2 ABOUT HERE

A decrease in surface area was observed, in the following order: F50:50B $>$ F50:50 $>$ F100 and F75:25. There was no difference in the specific surface area between the F75:25 and F100 formulations. Furthermore, spray-dried powders produced from LNC showed a decrease in surface area and pore volume compared with that prepared using water instead of the nanocapsules suspension.

Morphological characterization of the spray-dried lipid-core nanocapsules

Scanning electron microscopy (SEM) 
Fig. 3 shows SEM images of the spray-dried powders. All powders prepared from the LNC suspensions (F50:50, F75:25 and F100) were composed mainly of spherical particles that presented smoother surface and some invaginations. On the other hand, the powder prepared using water instead of the nanocapsule suspension (F50:50B) had particles with collapsed surface and more invaginations compared to formulations F50:50, F75:25, and F100.

INSERT FIGURE 3 ABOUT HERE

Confocal Raman microscopy

Single Raman afforded to obtain different spectra of L-leucine, maltodextrin, and LNC (Fig. 4).

\section{INSERT FIGURE 4 ABOUT HERE}

False color Raman images of F75:25 and F100 powders (Fig. 5A and 5B, respectively) shows only the yellow color, representing the LNC. On the other hand, false color images of the F50:50 formulation depicting the individual compounds in different colors $($ blue $=$ matrix of maltodextrin + leucine, yellow $=$ LNC) are presented in Fig. 6. Maltodextrin/leucine as adjuvants as well as LNC were successfully visualized forming bigger agglomerates (Fig. 6A) as well as smaller particles (Fig. 6B). 


\section{Determination of redispersion efficiency}

This analysis was carried out only for F50:50, since it was the only formulation that showed an easy aqueous redispersion profile by laser diffractometry. The particle size distribution profile of the redispersed F50:50 spray-dried formulation $(0.695 \mathrm{~g}$ in 5 $\mathrm{mL}$ of ultrapure water) is shown in Fig. 7.

\section{INSERT FIGURE 7 ABOUT HERE}

Fig. 7A shows the particle size distribution based on spherical equivalent diameter. Immediately after the redispersion $(0.695 \mathrm{~g}$ of powder in $5 \mathrm{~mL}$ of ultrapure water), four main particle populations were detected. The particle population with size distribution in the nanometer scale presents a profile that was quite similar to the distribution profile of the original LNC suspension. On the other hand, the particle size distribution analyzed in terms of numbers of particles (Fig. 7B) presented unimodal particle size distribution, with a profile that was similar to that of the original nanocapsule suspension.

Fig. 8 shows TEM images of the LNC original suspension (Fig. 8A) and the particles observed in the aqueous redispersion of the spray-dried powder F50:50 (Fig. 8B). Particles of both samples had similar morphological characteristics. 
Regarding the analysis of particle number density, the supernatant of the redispersed F50:50 spray-dried powder showed a value of $1.43 \times 10^{14}$. This value represents about $75 \%$ of the particle number density of the initial LNC suspension (1.93 $\left.\mathrm{x} 10^{14}\right)$.

\section{Discussion}

LNC suspensions were produced by interfacial deposition of a preformed polymer, poly( $\varepsilon$-caprolactone). LNC had mean diameter in the nanometric range and low polydispersity index, as expected for this kind of formulation formed by the diffusion of the organic solvent in the aqueous phase, followed by the solvent evaporation at the water/air interface (Fessi et al. 1989, Venturini et al. 2011).

The negative zeta potential close to the neutrality is in agreement with previous reports in the literature for LNC formulations (Venturini et al. 2011, Zanotto-Filho et al. 2013). Despite the inexpressive zeta potential module value, it is known that the presence of polysorbate 80 in LNC suspensions is able to prevent particle coalescence/aggregation due to a steric stabilization effect (Venturini et al. 2011).

LNC suspensions afford the advantage of encapsulating higher amounts of lipophilic drug over nanocapsules prepared with a single oil core (Jager et al. 2009, Venturini et al. 2011). Besides that, previous studies have described the advantages of the drug encapsulation in this type of polymeric nanoparticles, when compared to the non-encapsulated drug (Figueiro et al. 2013, Friedrich, et al. 2014, Frozza et al. 2013, Zanotto-Filho, et al. 2013). Therefore, this particle model was selected for this study.

According to the United States Pharmacopeia (2006), maltodextrin could be classified as an adjuvant freely soluble and readily dispersible in water meanwhile about 
lactose, an adjuvant commonly used as drying adjuvant to obtain powders containing lipid-core nanocapsules, was not any mention about its redispersion properties.

Besides that, in different studies conducted by Akhilesh and co-workers (2012) and Parikh and co-workers (2014), comparing the carriers used to produce proniosomes, maltodextrin is considering the most convenient adjuvant in comparison with sugars as sorbitol, manitol and lactose. Proniosomes are water-soluble carrier particles that are coated with surfactants and when hydrated as able to form niosomes, nonionic surfactant vesicles with potential applications to drug delivery of hydrophobic or amphiphilic drugs. It is important highlight that the rehydration process is mandatory to obtain the niosomes (Akhilesh et al. 2012). In a similar way, in our study the rehydration process of the powders is fundamental for guarantee the redispersion of the nanoparticles.

Taking these information together, we suggest that maltodextrin presents great potential as a drying adjuvant to obtain an easily redispersion of lipid-core nanocapsules from spray-dried formulations.

Because of the high hygroscopicity of maltodextrin, hydrophobic L-leucine was included in the formulation to avoid the formation of highly cohesive particles. This same strategy was used by Kho and co-workers (2010) to produce spray-dried antibiotic-loaded polycaprolactone nanoparticle aggregates using different sugars (mannitol and lactose) as drying adjuvants.

The concentrations and proportions between the adjuvants are established according previous studies published in the literature (Sou et al. 2013, Sou et al. 2015, Sou et al. 2011, Tewa-Tagne et al. 2007). 
Sou and co-workers (2013) concluded that combination of trehalose with 10 $20 \%$ of L-leucine appears to have a good potential for development into a universal carrier platform for delivery of biomacromolecules.

According to Kho and co-workers (2010), the leucine composition on the particle surface dictates the surface hydrophobicity of the aggregates of sugars and Lleucine, which in turn influences the particle wetting and consequently the aqueous redispersion process. Besides that, nanoparticles are known to compete with surfaceactive agents, such as L-leucine, to occupy the liquid-vapor interface upon dropolet evaporation.

Taking into account this information, we established the proportion of $10 \% \mathrm{~L}-$ leucine in the drying adjuvant mixture in order to avoid possible problems in the redispersion process of the lipid-core nanocapsules.

The drying process yields were between $64 \%$ and $68 \%$ for all spray-dried formulations containing LNC. These values are in agreement with spray-dried nanocapsules produced by different research groups using maltodextrin, lactose, mannitol (Hoffmeister et al. 2012, Tewa-Tagne et al. 2007), K30-PVP, hydroxypropylcellulose, hydroxypropylmethylcellulose (Tewa-Tagne et al. 2007), or Lleucine (Pourshahab et al. 2011) as drying adjuvants.

The addition of aqueous solution of drying adjuvants to NC suspensions before the spray-drying process is an important step to avoid solid/solid interactions between nanostructured carriers surface and the adjuvants, which may occur when they dissolve promptly in the LNC suspension (Tewa-Tagne et al. 2007). Furthermore, this procedure favors the homogeneity of the LNC + drying adjuvant dispersion. In this sense, the spray-dried powders produced in this study from diluted nanocapsules suspensions were 
prepared using drying adjuvants previously diluted in a predetermined volume of water, according to the composition of the formulations.

However, to the best of our knowledge, there is a lack of studies about the influence of the particle number density on the aqueous redispersion of spray-dried powders prepared from polymeric nanocapsules suspension. After their conversion in a solid dosage form intended for oral delivery, nanocapsules should redisperse in the gastrointestinal tract and therefore be reduced to similar particle sizes, providing the advantages of a nanosized drug delivery system. In this study, the influence of particle number density on powder redispersibility was evaluated using three different spraydried powders produced from LNC samples containing different particle number density values, in the following order: F100 $>$ F75:25>F50:50. The influence of particle number density was clearly observed from the particle size distributions obtained from laser diffraction analysis. The results indicate that the lower the number of particles in the sample fed to the spray-drying process, the better the particle size recovery after the aqueous redispersion. The recovery of particles matching the size of the initial particles was successful with a 50\% (v/v) concentration of nanocapsules (F50:50), pointing to the important advantages it offers when compared to the results of previous studies. TewaTagne and co-workers reported the best results of aqueous reconstitution for dried nanocapsules using a nanocapsule concentration of 1\% w/v (Tewa-Tagne et al. 2007). Furthermore, Hoffmeister and co-workers (2012) described spray-dried particles prepared with maltodextrin as a drying adjuvant and non-diluted nanocapsules, which recovery of particles at the nanoscale range required a 60 -min stirring run. In the present study, the recovery took less than $1 \mathrm{~min}$. This improved particle recovery from the spray-dried powder could be explained in light of to the presence of L-leucine in the spray-dried formulation and its influence on the surface tension (Gliniski et al. 2000). 
The addition of L-leucine to the nanocapsules aqueous suspension before the spraydrying process leads to a rapid decrease in surface tension of the system on the redispersion step, which promotes more efficient interactions between water and the hydrophilic matrix composed of maltodextrin making these step more efficient.

Moreover, L-leucine has been widely studied in the development of formulations intended for pulmonary delivery, due to its ability to improve a powder's aerodynamic characteristics, especially aerosolization properties (Ely et al. 2007). In order to test this hypothesis, a spray-dried powder was prepared without L-leucine (F50:50M). As observed in the particle size distribution, redispersion was not efficient, even after a 5-min stirring run, confirming our hypothesis.

Moreover, in order to go deeper in the understanding of these data, the influence of the surface area and pore volume of the spray-dried powders on the recovery of the nanoparticles after aqueous redispersion was evaluated. The values were compared to the spray-dried powder prepared with water instead of the nanocapsules suspension in order to evaluate the influence of the nanoparticles on their solid structure. All powders prepared from nanocapsules suspensions showed a decreased pore volume compared to the powder prepared without particles (F50:50B). This can be explained by the coating of the powder surface, as previously observed for nanoparticle-coated silicon-dioxide + diclofenac microparticles (Beck et al. 2006). However, these data do not point to the influence of pore volume on the redispersion behavior of the different spray-dried powders. SEM images confirmed that the presence of LNC alters the morphology of the spray-dried particles. F50:50B particles had a rugged surface, whereas F50:50, F75:25 and F100 particles were smoother and spherical. However, SEM revealed no differences in the surface morphology between these three formulations. On the other hand, surface area data showed that the formulation prepared with the sample containing the highest 
number of particles (F100) had the most significant decrease in surface area. This reduction was in the following order: F100 $>$ F75:25 $>$ F50:50. These finding points to the influence of particle number density of the sample fed to the spray-drying process on redispersion behavior. The influence of the presence of polymeric nanoparticles on the surface area of nanoparticle-coated organic-inorganic microparticles was previously reported (Beck et al. 2008), but the influence of particle number density is first being reported in the present study. The influence of the surface area of powders on their nanoparticle recovery could be explained by the different number of nanocapsules on the surface of the powder, which influences water diffusion rate and, consequently, the dissolution of the drying adjuvant, making redispersion more difficult. It was hypothesized that the powders prepared with the higher number of polymeric nanocapsules (F100 and F75:25) would coat all or almost all their particle surface, whereas the powder prepared with the sample containing the lowest number of particles (F50:50) would also coat the particle surface, but partially, facilitating water uptake, solubilization of the drying adjuvant, and redispersion of the particles. However, this hypothesis could not be confirmed by SEM analyses, as discussed above.

In this scenario, Confocal Raman Microscopy (CRM) analysis was carried out. This technique has been used to analyze different carrier systems, ranging from solid dispersion and tablets up to drug-eluting coatings and polymeric microparticles (Smith et al. 2015). It has been successfully used for visualizing dexamethasone homogeneous distribution in submicron polymeric particles (Beber et al. 2014), and for chemically selective visualization of particle uptake into human buccal epithelial cells (Kann et al. 2014). In this study, CRM enabled the visualization of the spatial distribution of the different compounds (chemically selective and spatially resolved) in the spray-dried powders, affording to better understand the influence of the particle number density of 
the fed sample on the redispersion behavior of such powders. For formulations F75:25 and F100, LNC were visualized on the surface of the powder agglomerates with no sign of the drying adjuvants, confirming the complete coating of the drying adjuvants by the nanocapsules when the samples with the higher particle number density were used to prepare them. However, in the F50:50 formulation, maltodextrin/leucine and LNC could be visualized at different locations in the agglomerates, showing that surface of powders was not completely coated by the polymeric nanocapsules. This confirms our previous hypothesis about the correlation between compound distribution and good redispersion characteristics of these spray-dried powders. Although we have an indicative of an ideal number of particles for the production of the spray-dried lipid-core nanocapsules formulation, under the conditions and with the drying adjuvants used in this study, it is not possible to accurately estimate the exact number of lipid-core nanocapsules in suspension required to completely coat the surface of the particles of the powders by conventional available methods. Further studies are needed to quantitatively and accurately correlate the exactly number of particles that limits the good performance of the redispersion of nanocapsules from dried formulations. Moreover, it may be suggested that, for formulation F50:50, the presence of the drying adjuvants on the particles surfaces acts like spacers between the nanocapsules, improving water diffusion in the spray-dried powder and facilitating redispersion into the initial nanocapsules.

Finally, the good recovery of nanoparticles from the best formulation (F50:50) was confirmed under the conditions simulating its dispersion to the original volume ( $0.695 \mathrm{~g}$ of powder in $5 \mathrm{~mL}$ of ultrapure water). It is important to highlight that this powder mass $(0.695 \mathrm{~g})$ was chosen because it would allow the recovery of the initial particle number (as measured in the NC suspension before the spray-drying process). The particle size distribution profile obtained by laser diffraction analyses had four main 
particle size populations. The population with a particle size distribution in the nanometer scale presents a very similar profile to the distribution profile of the original LNC suspension. Previous studies evaluated the aqueous redispersion of small amounts of powder with larger volumes of water, but they did not find any correlation with the original volume of the fed sample. Furthermore, a considerable number of protocols employ high temperature, surfactants or an ultrasonic bath to enable the redispersion of nanoparticles (Kaye et al. 2009, Li et al. 2016, Schafroth et al. 2012, Sinsuebpol et al. 2013, Tomoda et al. 2009, Tomoda et al. 2008). However, in the present study the dilution of $0.695 \mathrm{~g}$ of F50:50 powder in a 5-mL volumetric flask followed by vortexing for $2 \mathrm{~min}$ at room temperature clearly shows that redispersing the spray-dried powder could be a very simple process, since some parameters have been taken into account, like the particle density number of the sample fed to the spray-dryer. Moreover, the redispersed powder (F50:50) had a unimodal particle size distribution, when analyzed on number basis, with a distribution profile that is similar to that of the original nanocapsule suspension. This result indicates that the contribution of the micrometric populations observed for the redispersed F50:50 powder can be considered minimal. Furthermore, TEM analyses were carried to observe if any change on the particles morphology could be seen after the aqueous redispersion of this powder (F50:50). The recovered nanoparticles kept their original morphological characteristics after the spraydrying step, since no changes were observed. In addition, the estimated number of particles per unit of volume $\left(\mathrm{N} \mathrm{cm}^{-3}\right)$ after the redispersion of this powder corresponds to $75 \%$ of estimated number of particles per unit of volume for the original LNC suspension used as the starting suspension (fed sample) in the spray-drying process. However, we hypothesized that the rest of the particles $(25 \%)$ still remain as micrometric aggregates, as can be observed in Fig. 7A. 


\section{Conclusions}

The influence of the particle number density of the nanocapsule suspension fed in the spray-drying process on the redispersion profiles of the corresponding powders prepared with water-soluble drying adjuvants was established. The use of samples with higher number of particles led to a complete coating of the powders surfaces, making redispersion difficult. An easily redispersible spray-dried LNC powder was produced by spray-drying using water-soluble drying adjuvants and a fed sample with a particle number density of $0.387 \times 10^{13}$ LNC per milliliter. The correct choice of the adjuvants, such as L-leucine and maltodextrin, acting as spacers between the nanocapsules, besides the selection of the appropriate number density of nanoparticles from the lipid-core nanocapsules suspension, avoiding the complete covering of the microaggregates were fundamental to produce spray-dried powders with good recovery properties. These results highlight the needing of an optimization of the initial particle number density in the liquid formulation to develop redispersible spray-dried LNC powders. Moreover, this study represents a promising approach to the development of easily redispersible spray-dried LNC formulations intended for oral administration.

\section{Acknowledgements}

This work was supported by Coordination for the Improvement of Higher Education Personnel - CAPES, Brazil, National Council for Scientific and Technological CNPq/MCTI, Research Support Foundation of Rio Grande do Sul - FAPERGS, Brazil and the German Academic Exchange Service - DAAD, Germany. 


\section{Declaration of interest}

The authors report no conflict of interest. 


\section{References}

Abdewahed W, Degobert D, Stainmesse S, Fessi H. 2006. Freeze-drying of nanoparticles: formulation, process and storage considerations. Advanced Drug Delivery Reviews. 38:16881713.

Akhilesh D, Faishal G, Kamath JV. 2012. Comparative study of carriers used in proniosomes. International Journal of Pharmaceutical and Chemical Sciences.1:164-173.

Arpagaus C, John P, CollenberG A, Rütti D. 2017. Nanocapsules formation by nano spray drying. In: Nanoencapsulation Technologies for the Food and Neutraceutical Industries. London: Elsevier Academic Press. p. 346-401.

Beber TC, Andrade DF, Kann B, Fontana MC, Coradini K, Windbergs M, Beck RC. 2014. Submicron polymeric particles prepared by vibrational spray-drying: Semisolid formulation and skin penetration/permeation studies. European Journal of Pharmaceutics and Biopharmaceutics. 88:602-613.

Beck RC, Pohlmann AR, Hoffmeister C, Gallas MR, Collnot E, Schaefer UF, Guterres SS, Lehr CM. 2007. Dexamethasone-loaded nanoparticle-coated microparticles: correlation between in vitro drug release and drug transport across Caco-2 cell monolayers. European Journal of Pharmaceutics and Biopharmaceutics. 67:18-30.

Beck RCR, E. HS, Guterres SR, Ré MI, Benvenutti EV, Pohlmann AR. 2006. Nanoparticlecoated Organic-Inorganic Microparticles: Experimental Design and Gastrointestinal Tolerance Evaluation. Química Nova. 29:990-996.

Beck RCR, Lionzo MIZ, Costa TMH, Benvenutti EV, Ré MI, Gallas MR, Pohlmann AR, Guterres SR. 2008. Surface morphology of spray-dried nanoparticle-coated microparticles designed as an oral drug delivery system. Brazilian Journal of Chemical Engineering. 25:389398.

Beck RCR, Chaves PS, Goyanes A, Vukosavlkevic B, Buanz A, Windbergs M, Basit AW, Gaisford S. 2017. 3D printed tablets loaded with polymeric nanocapsules: An innovative approach to produce customized drug delivery systems. International Journal of Pharmaceutics. 528:268-279.

Bueno K, Adorne MD, Jornada DS, da Fonseca FN, Guterres SS, Pohlmann AR. 2013. In vivo gastroprotective effect of nanoparticles: influence of chemical composition and volume fraction. Current Pharmaceutical Design.19:7294-7300.

Chasteigner S, Cavé G, Fessi H, Devissaguet J, Puisieux F. 1996. Freeze-drying of itraconazoleloaded nanosphere suspensions: a feasibility study. Drug Development Research. 38:116-124. 
Dimer FA, Ortiz M, Pase CS, Roversi K, Friedrich RB, Pohlmann AR, Burger ME, Guterres SS. 2014. Nanoencapsulation of olanzapine increases its efficacy in antipsychotic treatment and reduces adverse effects. Journal of Biomedical Nanotechnology. 10:1137-1145.

El-Sherbiny IM, Smyth HD. 2010. Biodegradable nano-micro carrier systems for sustained pulmonary drug delivery: (I) self-assembled nanoparticles encapsulated in respirable/swellable semi-IPN microspheres. International Journal of Pharmaceutics. 395:132-141.

Ely L, Roa W, Finlay WH, Lobenberg R. 2007. Effervescent dry powder for respiratory drug delivery. European Journal of Pharmaceutics and Biopharmaceutics. 65:346-353.

Fessi H, Puisieux F, Devissaguet J, Ammoury N, Benita S. 1989. Nanocapsules formation by interfacial deposition following solvent displacement. International Journal of Pharmaceutics. 55:R1-R4.

Fiel LA, Rebêlo LM, Santiago TM, Adorne MD, Guterres SS, Sousa JS, Pohlmann AR. 2011. Diverse deformation properties of polymeric nanocapsules and lipid-core nanocapsules. Soft Matter. 7:7240-7247.

Figueiro F, Bernardi A, Frozza RL, Terroso T, Zanotto-Filho A, Jandrey EH, Moreira JC, Salbego CG, Edelweiss MI, Pohlmann AR, et al. 2013. Resveratrol-loaded lipid-core nanocapsules treatment reduces in vitro and in vivo glioma growth. Journal of Biomedical Nanotechnology. 9:516-526.

Fontana MC, Rezer JF, Coradini K, Leal DB, Beck RC. 2011. Improved efficacy in the treatment of contact dermatitis in rats by a dermatological nanomedicine containing clobetasol propionate. European Journal of Pharmaceutics and Biopharmaceutics. 79:241-249.

Friedrich RB, Fontana MC, Bastos MO, Pohlmann AR, Guterres SS, Beck, RCR. 2010. Drying Polymeric Drug-Loaded Nanocapsules: The Wet Granulation Process as a Promising Approach. Journal of Nanoscience and Nanotechnology. 10:616-621.

Friedrich RB, Dimer FA, Guterres SS, Beck RC, Pohlmann AR. 2014. Nanoencapsulation of tacrolimus in lipid-core nanocapsules showed similar immunosuppressive activity after oral and intraperitoneal administrations. Journal of Biomedical Nanotechnology. 10:1599-1609.

Frozza RL, Bernardi A, Hoppe JB, Meneghetti AB, Battastini AM, Pohlmann AR, Guterres SS, Salbego C. 2013a. Lipid-core nanocapsules improve the effects of resveratrol against Abetainduced neuroinflammation. Journal of Biomedical Nanotechnology. 9:2086-2104.

Frozza RL, Bernardi A, Hoppe JB, Meneghetti AB, Matte A, Battastini AM, Pohlmann AR, Guterres SS, Salbego C. 2013b. Neuroprotective effects of resveratrol against Abeta administration in rats are improved by lipid-core nanocapsules. Molecular Neurobiology. 47:1066-1080.

Gliniski J, Chavepeyer G, Platten JK. 2000. Surface properties of aqueous solutions of Lleucine. Biophysical Chemistry. 84:99-103. 
Guterres SS, Beck, RCR, Pohlmann, AR. 2009.Spray-drying technique to prepare innovative nanoparticulated formulations for drug administration: a brief overview. Brazilian Journal of Physics. 39:205-209.

Hoffmeister CR, Durli TL, Schaffazick SR, Raffin RP, Bender EA, Beck RC, Pohlmann AR, Guterres SS. 2012. Hydrogels containing redispersible spray-dried melatonin-loaded nanocapsules: a formulation for transdermal-controlled delivery. Nanoscale Research Letters. 7:251-263.

Jager E, Venturini CG, Poletto FS, Colome LM, Pohlmann JP, Bernardi A, Battastini AM, Guterres SS, Pohlmann AR. 2009. Sustained release from lipid-core nanocapsules by varying the core viscosity and the particle surface area. Journal of Biomedical Nanotechnology. 5:130140 .

Jaques JA, Doleski PH, Castilhos LG, da Rosa MM, Souza Vdo C, Carvalho FB, Marisco P, Thorstenberg ML, Rezer JF, Ruchel JB, et al. 2013. Free and nanoencapsulated curcumin prevents cigarette smoke-induced cognitive impairment and redox imbalance. Neurobiology of Learning and Memory. 100:98-107.

Kann B, Teubl BJ, Roblegg E, Windbergs M. 2014. Label-free in vitro visualization of particle uptake into human oral buccal epithelial cells by confocal Raman microscopy. Analyst. 139:5069-5074.

Kaye RS, Purewal TS, Alpar HO. 2009. Simultaneously manufactured nano-in-micro (SIMANIM) particles for dry-powder modified-release delivery of antibodies. Journal of Pharmaceutical Sciences. 98:4055-4068.

Lebhardt T, Roesler S, Uusitalo HP, Kissel T. 2011. Surfactant-free redispersible nanoparticles in fast-dissolving composite microcarriers for dry-powder inhalation. European Journal of Pharmaceutics and Biopharmaceutics. 78:90-96.

Li H, Dong WF, Zhou JY, Xu XM, Li FQ. 2013. Triggering effect of N-acetylglucosamine on retarded drug release from a lectin-anchored chitosan nanoparticles-in-microparticles system. International Journal of Pharmaceutics. 449:37-43.

Li M, Lopez N, Bilgili E. 2016. A study of the impact of polymer-surfactant in drug nanoparticle coated pharmatose composites on dissolution performance. Advanced Powder Technology. 27:1625-1636.

Mohajel N, Najafabadi AR, Azadmanesh K, Vatanara A, Moazeni E, Rahimi A, Gilani K. 2012. Optimization of a spray drying process to prepare dry powder microparticles containing plasmid nanocomplex. International Journal of Pharmaceutics. 423:577-585.

Morgen M, Bloom C, Beyerinck R, Bello A, Song W, Wilkinson K, Steenwyk R, Shamblin S. 2012. Polymeric nanoparticles for increased oral bioavailability and rapid absorption using celecoxib as a model of a low-solubility, high-permeability drug. Pharmaceutical Research. 29:427-440. 
Muller CR, Bassani VL, Pohlmann AR, Michalowski CB, Petrovick PR, Guterres SS. 2000. Preparation and characterization of spray-dried polymeric nanocapsules. Drug Development and Industrial Pharmacy. 26:343-347.

Muttil P, Pulliam B, Garcia-Contreras L, Fallon JK, Wang C, Hickey AJ, Edwards DA. 2010. Pulmonary immunization of guinea pigs with diphtheria CRM-197 antigen as nanoparticle aggregate dry powders enhance local and systemic immune responses. The AAPS Journal. 12:699-707.

Okuyama K, Abdullah M, Lenggoro W, Iskandar F. 2006. Preparation of functional nanostructured particles by spray drying. Advanced Powder Technology. 17:587-611.

Ozeki T, Beppu S, Mizoe T, Takashima Y, Yuasa H, Okada H. 2006. Preparation of polymeric submicron particle-containing microparticles using a 4-fluid nozzle spray drier. Pharmaceutical Research. 23:177-183.

Pamujula S, Graves RA, Freeman T, V. S, Bostanian LA, Kishore V, Mandal TK. 2004. Oral delivery of spray dried PLGA/amifostine nanoparticles. Journal of Pharmacy and Pharmacology. 2004 :56:1119-1125.

Pohlmann AR, Weiss V, Mertins O, Silveira NP, Guterres SS. 2002. Spray-dried indomethacinloaded polyester nanocapsules and nanospheres: development, stability evaluation and nanostructure models. European Journal of Pharmaceutical Sciences. 16:305-312.

Poletto FS, Jager E, Cruz L, Pohlmann AR, Guterres SR. 2008. The effect of polymeric wall on the permeability of drug-loaded nanocapsules. Materials Science and Engineering: C. 28:472478.

Pourshahab PS, Gilani K, Moazeni E, Eslahi H, Fazeli MR, Jamalifar H. 2011. Preparation and characterization of spray dried inhalable powders containing chitosan nanoparticles for pulmonary delivery of isoniazid. Journal of Microencapsulation. 28:605-613.

Rigo LA, Frescura V, Fiel L, Coradini K, Ourique AF, Emanuelli T, Quatrin A, Tedesco S, da Silva CB, Guterres SS, Pohlmann AR, Beck RCR. 2014. Influence of the type of vegetable oil on the drug release profile from lipid-core nanocapsules and in vivo genotoxicity study. Pharmaceutical Development and Technology. 19:789-798.

Schaffazick SR, Guterres SS, Pohlmann AR, Lucca FL. 2003. Caracterização e estabilidade físico-química de sistemas poliméricos nanoparticulados para administração de fármacos. Química Nova. 26:726 - 737.

Schaffazick SR, Pohlmann AR, Mezzalira G, Guterres SS. 2006. Development of nanocapsule suspensions and nanocapsule spray-dried powders containing melatonin. Journal of Brazilian Chemical Society. 17:562-569.

Schafroth N, Arpagaus C, Jadhav UY, Makne S, Douroumis D. 2012. Nano and microparticle engineering of water insoluble drugs using a novel spray-drying process. Colloids and Surfaces B, Biointerfaces. 90:8-15. 
Sham JO, Zhang Y, Finlay WH, Roa WH, Lobenberg R. 2004. Formulation and characterization of spray-dried powders containing nanoparticles for aerosol delivery to the lung. International Journal of Pharmaceutics. 269:457-467.

Sinsuebpol C, Chatchawalsaisin J, Kulvanich P. 2013. Preparation and in vivo absorption evaluation of spray dried powders containing salmon calcitonin loaded chitosan nanoparticles for pulmonary delivery. Drug Design, Development and Therapy. 7:861-873.

Smith GP, McGoverin CM, Fraser SJ, Gordon KC. 2015. Raman imaging of drug delivery systems. Advanced Drug Delivery Reviews. 89:21-41.

Sou T, Kaminskas LM, Nguyen TH, Carlberg R, McIntosh MP, Morton DA. 2013. The effect of amino acid excipients on morphology and solid-state properties of multi-component spraydried formulations for pulmonary delivery of biomacromolecules. European Journal of Pharmaceutics and Biopharmaceutics. 83:234-243.

Sou T, Morton DA, Williamson M, Meeusen EN, Kaminskas LM, McIntosh MP. 2015. SprayDried Influenza Antigen with Trehalose and Leucine Produces an Aerosolizable Powder Vaccine Formulation that Induces Strong Systemic and Mucosal Immunity after Pulmonary Administration. Journal of Aerosol Medicine and Pulmonary Drug Delivery. 28:361-371.

Sou T, Orlando L, McIntosh MP, Kaminskas LM, Morton DA. 2011. Investigating the interactions of amino acid components on a mannitol-based spray-dried powder formulation for pulmonary delivery: A design of experiment approach. International Journal of Pharmaceutics. 421:220-229.

Tewa-Tagne P, Briancon S, Fessi H. 2007. Preparation of redispersible dry nanocapsules by means of spray-drying: development and characterisation. European Journal of Pharmaceutical Sciences. 30:124-135.

Tomoda K, Ohkoshi T, Hirota K, Sonavane GS, Nakajima T, Terada H, Komuro M, Kitazato K, Makino K. 2009. Preparation and properties of inhalable nanocomposite particles for treatment of lung cancer. Colloids and surfaces B, Biointerfaces. Jul 1;71:177-182.

Tomoda K, Ohkoshi T, Nakajima T, Makino K. 2008. Preparation and properties of inhalable nanocomposite particles: effects of the size, weight ratio of the primary nanoparticles in nanocomposite particles and temperature at a spray-dryer inlet upon properties of nanocomposite particles. Colloids and Surfaces B, Biointerfaces. 64:70-76.

Ungaro F, d'Angelo I, Coletta C, d'Emmanuele di Villa Bianca R, Sorrentino R, Perfetto B, Tufano MA, Miro A, La Rotonda MI, Quaglia F. 2012. Dry powders based on PLGA nanoparticles for pulmonary delivery of antibiotics: modulation of encapsulation efficiency, release rate and lung deposition pattern by hydrophilic polymers. Journal of Controlled Release. 157:149-159. 
Venturini CG, Jager E, Oliveira CP, Bernardi A, Battastini AM, Guterres SR, Pohlmann AR. 2011. Formulation of lipid core nanocapsules. Colloids and Surfaces A: Physicochemical and Engineering Aspects. 375:200-208.

Zanotto-Filho A, Coradini K, Braganhol E, Schroder R, de Oliveira CM, Simoes-Pires A, Battastini AM, Pohlmann AR, Guterres SS, Forcelini CM, et al. 2013. Curcumin-loaded lipidcore nanocapsules as a strategy to improve pharmacological efficacy of curcumin in glioma treatment. European Journal of Pharmaceutics and Biopharmaceutics. 83:156-167.

Zattoni A, Loli Piccolomini E, Torsi G, Reschiglian P. 2003. Turbidimetric detection method in flow-assisted separation of dispersed samples. Analytical Chemistry. 75:6469-6477. 


\section{Figure captions:}

Fig. 1. Particle size distribution profile of different candidates for redispersible spraydried lipid-core nanocapsules formulation (F100, F75:25, F50:50) and original lipidcore nanocapsules suspension (LNC-B) obtained by laser diffraction directly after production.

Fig. 2. Particle size distribution profile of the F50:50M (without L-leucine) formulation obtained by laser diffractometry, before $(0 \mathrm{~min})$ (immediately after addition of the sample into the analyzer compartment) and after 5 min of stirring.

Fig. 3. SEM images of F50:50B (A), F50:50 (B), F75:25 (C), and F100 (D) spray-dried formulations under 20,000 x magnification.

Fig. 4. Single Raman spectra of individual compounds: leucine, maltodextrin, and LNC. Identification bands are highlighted.

Fig. 5. False color Raman images of F75:25 (A) and F100 (B) agglomerates. LNC are depicted in yellow.

Fig. 6. False color Raman images of the F50:50 formulation. (A) A large representative agglomerate, and (B) smaller particles. Maltodextrin and leucine are depicted in blue, while LNC are depicted in yellow.

Fig. 7. Particle size distribution profile of the F50:50 spray-dried formulation after aqueous redispersion ( $0.695 \mathrm{~g}$ in $5 \mathrm{~mL}$ of ultrapure water) obtained by laser diffraction at 0 min (immediately after addition of the sample into the analyzer compartment). (A) Particle diameter expressed by volume of particles and (B) particle diameter expressed as number of particles $(n=3)$.

Fig. 8. TEM images of (A) LNC original suspension and (B) redispersed F50:50 (0.695 $\mathrm{g}$ in $5 \mathrm{~mL}$ of water) under $250,000 \mathrm{x}$ magnification. 